\title{
Sensor Optimization Selection Based on Fault Detectability and Trackability
}

\author{
Luo Jianlu, Tan Xiaodong \\ Officers College of PAP, Chendu610213, China \\ xdt_nudt37@163.com
}

\begin{abstract}
Keywords: Health monitoring; Sensor Optimization Selection Model (SOSM); Adaptive Simulated Annealing Genetic Algorithm (ASAGA)

Abstract. Correctly selecting and reasonably arranging sensors are critical to high fidelity health assessment and low testing costs. A novel approach of sensor optimization placement for health monitoring based on fault detectability and trackability is proposed in this paper. Firstly, the requirements of sensor selection for health monitoring, the definitions and calculations of fault detectability and trackability are presented. Thus, a Sensor Optimization Selection Model (SOSM), whose objectives are to maximize the fault detectability and trackability and minimize cost of sensors, is built. Afterwards, an Adaptive Simulated Annealing Genetic Algorithm (ASAGA) is implemented to solve the SOSM. Finally, the real gearboxes and experimental data are used to verify the effectiveness of the SOSM proposed in this paper and its solution. The results from this study have shown that the approach can provide a better strategy for health monitoring in order to reduce the test cost, improve the reliability and the capability.
\end{abstract}

\section{Introduction}

Sensor data provide the foundation for health monitoring of most complex systems ${ }^{[1-3]}$. In practice, excess sensors will ultimately reduce systems' reliability and increase the monitoring cost. But if sensors at a very small number are used to save a lot of money or effort, it will directly result in the decrease in health estimation accuracy or the failure to achieve the objectives of health monitoring. Obviously, a tradeoff should be made between the number of sensors and the financial cost. Therefore, sensor selection/placement plays an important role in designing an effective health monitoring system.

Therefore, to better improve those performance levels of fault prognostics and health assessment, this paper quantifies the fault detectability and trackability of a sensor, thus a Senor Optimization Selection Model (SOSM), which maximizes the fault detectability and trackability of the system to decrease the cost of sensors as soon as possible on the basis of meeting the scheduled testability requirements. Finally, an experimental data provided by Mechanical Diagnosis Test Bed (MDTB) of Applied Research Laboratory (ARL) at Pennsylvania State University are used to verify the effectiveness of the method proposed in this paper. The main purpose of this paper is to provide a straightforward sensor optimization placement procedure to provide more useful information for health monitoring, fault prognostics and health assessment.

\section{The SOSM for health monitoring}

Correct selection and reasonable arrangement of sensors is critical to achieve high fidelity system health assessment and low testing costs ${ }^{[4-5]}$. Actually, detecting the incipient fault and tracking the fault evolution process directly affect the precision and validation of fault prognostics and health assessment. Thus, for sensor selection of health monitoring, the selected sensors should contain abundance of health information in order to detect the incipient fault as early as possible and effectively track the fault growth or evolution process ${ }^{[2]}$. The primary task of sensor selection of health monitoring is to compare the abilities of all sorts of sensors to detect the incipient fault and track the fault evolution process. 
So, the fault detectability and trackability of sensors need to be defined and quantified in order to provide data support for sensor selection of health monitoring.

The related definitions of a SOSM are described as follows:

(1) The available sensors in a gearbox system $\mathrm{SS}=\left\{\mathrm{ss}_{1}, \mathrm{Ss}_{2}, \ldots, \mathrm{Ss}_{j}, \ldots, \mathrm{Ss}_{N}\right\}$, where $N$ is the number of sensors;

(2) The fault mode set $\mathrm{F}=\left\{f_{1}, f_{2}, \ldots, f_{i}, \ldots, f_{M}\right\}$, where $M$ is the number of fault modes;

(3) $\mathrm{SST}_{i j}$ and $\mathrm{SSD}_{i j}$ are the trackability and detectability of $\mathrm{ss}_{j}$ for fault $f_{i}$, which can be calculated in Tan at al. ${ }^{[6]}$, respectively.

According to the requirements of SOSM for gearbox health monitoring, the paper proposes an optimal model which maximizes the fault trackability and minimizes the test cost based on fault detectability and trackability of sensors and with a constraint that FDR and FIR are greater than FDR* and FIR* ${ }^{[7]}$. The objective function of the optimal model is defined as follows:

$$
\left\{\begin{array}{l}
S S^{*}=\underset{S S}{\arg }\left\{\max \left(\sum_{i} \sum_{j} S S T_{i j}\right) \cup \max \left(\sum_{i} \sum_{j} S S D_{i j}\right) \cup \min \left(\sum C\left(S s_{i}\right)\right)\right\} \\
\text { s.t. } F D R \geq F D R^{*}, F I R \geq F I R^{*} .
\end{array}\right.
$$

where, SS is the available sensor set in the system. SS* is the optimal sensor set. $\mathrm{SST}_{i j}$ and $\mathrm{SSD}_{i j}$ are the trackability and detectability of $\mathrm{ss}_{j}$ for fault $f_{i}$. and $\mathrm{C}\left(\mathrm{ss}_{j}\right)$ is the cost of sensor $\mathrm{ss}_{j}$. FDR, FIR are the Fault Detection Rate and Fault Isolation Rate of the gearbox after selecting an optimal sensor, respectively. FDR*, and FIR* are testability indies that a system need to satisfy, respectively.

\section{Case study}

The experimental data are provided by the Applied Research Laboratory at Pennsylvania State University on three test runs of single reduction helical gearboxes ${ }^{[8]}$, which are named as TR\#5, TR\#10 and TR\#12, respectively. In three test runs, only the gears of TR\#5 are subject to obvious damage, i.e., two adjacent broken teeth $(40,41)$ and one cracked tooth $(44)$ of an output gear are found after test rig shutdown. So, TR\#5 is used to validate the proposed method in this paper. Gearbox information and the test run time specifications of TR\#5 can refer to Miao ${ }^{[9]}$. The total number of running hours is $127.4 \mathrm{~h}$, which includes 83 data files, and the relationship between the timestamp report and the file number is introduced by Wang et $\mathrm{al}^{[10]}$. Nine accelerometers (e.g.A02-A07, A10-A12) are placed on the test bed, and six of these are single-axis, shear-type with a bandwidth of $20 \mathrm{kHz}$ and the other three accelerometers are a triaxial, shear-type with a bandwidth of $8 \mathrm{kHz}$. Moreover, a total of 25 thermocouples (e.g. T04-T27, T31) are available for temperature readings on the MDTB. The thermocouples cover the gearbox and provide a low resolution image of surface temperature. The attributions of 34 sensors are listed in Table 1.

Table 1 Sensor Attributions

\begin{tabular}{|c|c|c|c|}
\hline Sensor Codes & Sensor Names & Cost $(\$)$ & TTD \\
\hline A02-A07 & Single axis accelerometer sensor & 200 & $2 \mathrm{~ms}$ \\
\hline A10,A11,A12 & Triaxial accelerometer & 200 & $2 \mathrm{~ms}$ \\
\hline T04,T26 & Magnet mount thermocouples & 100 & $20 \mathrm{~ms}$ \\
\hline Sensor Codes & Sensor Names & Cost $(\$)$ & TTD \\
\hline T05,T07,T17,T25 & Probe thermocouples & 100 & $20 \mathrm{~ms}$ \\
\hline T06,T08-T16,T18-T24,T27,T31 & Adhesive thermocouples & 100 & $20 \mathrm{~ms}$ \\
\hline
\end{tabular}

In military and commercial applications of gearboxes, the development of better health monitoring for maintenance practice is driven by the desire to reduce the risk of catastrophic failures, minimize maintenance costs, maximize system availability, and increase platform reliability. Obviously, redundant sensors will increase the test cost and decrease the system reliability. So the main objective of this paper is to select the optimal sensors according to their detectability and trackability for tooth damages on the basis of meeting the scheduled testability indices. For the requirements of health monitoring, the testability indices of the gearbox are assumed as: FDR $>98 \%$, FIR $>94 \%$. 
By using the SOSM method introduced in Section II, the detailed modeling process of SOSM is presented. Through integrating structural and functional information of the gearbox, the FS dependency of gearboxes is obtained and its partial contents are listed in Table 2.

Table 2 The FS dependency of gearboxes

\begin{tabular}{|c|l|l|c|c|c|c|c|c|c|c|c|c|c|}
\hline \multicolumn{9}{|c|}{ Fault modes } & \multicolumn{10}{c|}{ Sensor set } \\
\hline No & \multicolumn{1}{|c|}{ Names } & Fault Rate & A02 & A03 & A04 & A06 & A07 & A10 & A11 & A12 & $\ldots$ & T25 & T26 \\
\hline$f_{1}$ & $\begin{array}{l}\text { Inter-turn short } \\
\text { circuit }\end{array}$ & $1.0 \times 10^{-6}$ & 0 & 0 & 0 & 0 & 0 & 0 & 0 & 0 & $\ldots$ & 0 & 0 \\
\hline$f_{2}$ & Broken rotor-bar & $3.5 \times 10^{-6}$ & 0 & 0 & 0 & 0 & 0 & 0 & 0 & 0 & $\ldots$ & 0 & 0 \\
\hline$f_{3}$ & Bearing damage & $4.1 \times 10^{-6}$ & 0 & 0 & 0 & 1 & 1 & 0 & 0 & 0 & $\ldots$ & 0 & 0 \\
\hline$f_{4}$ & Misaligned motor & $0.3 \times 10^{-6}$ & 0 & 0 & 0 & 1 & 1 & 0 & 0 & 0 & $\ldots$ & 0 & 0 \\
\hline$f_{5}$ & skidding & $0.09 \times 10^{-6}$ & 0 & 0 & 0 & 0 & 0 & 0 & 0 & 0 & $\ldots$ & 0 & 0 \\
\hline$f_{6}$ & Driven gear crack & $4.6 \times 10^{-6}$ & 1 & 1 & 1 & 0 & 0 & 0 & 0 & 0 & $\ldots$ & 1 & 1 \\
\hline$f_{7}$ & Pinion gear crack & $4.2 \times 10^{-6}$ & 0 & 0 & 0 & 0 & 0 & 1 & 1 & 1 & $\ldots$ & 0 & 0 \\
\hline
\end{tabular}

For each sensor, indirect and signal-based sensing is influenced by noise from the surrounding environment. In order to extract useful features including more tooth damage information from the background noise, many frequency and time-frequency domain methods have been developed primarily for vibratory response signals. In this paper, the Fault Growth Parameter (FGP) proposed by Miao is used to build the fault evolution curves described by all sensors ${ }^{[8]}$. The evolution curves described by accelerometers A02, A03 and A04 are shown in Fig. 2(a). As shown, the tooth damage index changes un-obviously from file 1 to 12 . But, at the file 13, the damage index increases abruptly which means the incipient fault occurs. For the three sensors, their fault evolution curves present a monotonic increasing at a whole.

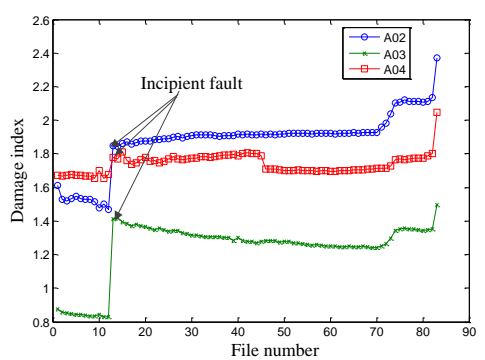

(a)

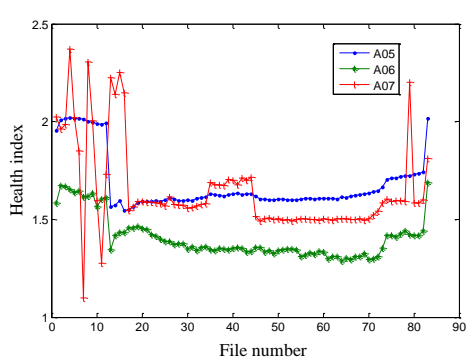

(b)

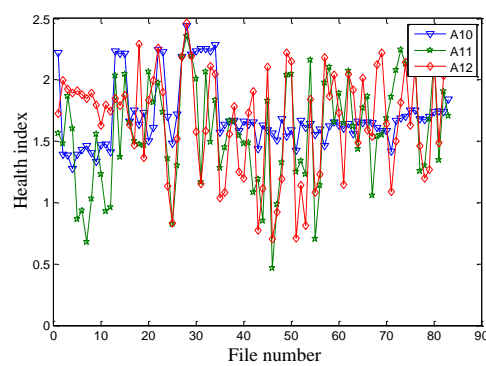

(c)

Fig. 1 The comparisons of fault evolution curves described by night sensors

In Fig. 1(b), the fault evolution trends described by accelerometers A05, A06 and A07 are shown, and Fig. 1(c) shows the fault evolution trends described by accelerometers A11, A12 and A13. For saving space, this paper only lists the fault evolution curves of tooth damage described by nine accelerometers.

In order to quantify the detectability and trackability of all sensors equipped in the gearbox based on fault evolution curves described by these sensors, SNR of all sensors are assumed 15 in this paper, thus their detectability and trackability for each fault can be calculated, and the partial results are listed in Table 3 and 4, respectively.

Table 3 The fault detectability of sensors $(S S D)$

\begin{tabular}{|c|c|c|c|c|c|c|c|c|c|c|c|c|}
\hline & $\mathrm{A} 02$ & $\mathrm{~A} 03$ & $\mathrm{~A} 04$ & $\mathrm{~A} 05$ & $\mathrm{~A} 06$ & $\mathrm{~A} 07$ & $\mathrm{~A} 10$ & $\mathrm{~A} 11$ & $\mathrm{~A} 12$ & $\ldots$ & $\mathrm{T} 25$ & $\mathrm{~T} 26$ \\
\hline$f_{1}$ & 0 & 0 & 0 & 0 & 0 & 0 & 0 & 0 & 0 & $\ldots$ & 0 & 0 \\
\hline$f_{2}$ & 0 & 0 & 0 & 0 & 0 & 0 & 0 & 0 & 0 & $\ldots$ & 0 & 0 \\
\hline$f_{3}$ & 0 & 0 & 0 & 0 & 0.0001 & 0.0022 & 0 & 0 & 0 & $\ldots$ & 0 & 0 \\
\hline$f_{4}$ & 0 & 0 & 0 & 0 & 0.0001 & 0.0022 & 0 & 0 & 0 & $\ldots$ & 0 & 0 \\
\hline$f_{5}$ & 0 & 0 & 0 & 0 & 0 & 0 & 0 & 0 & 0 & $\ldots$ & 0 & 0 \\
\hline$f_{6}$ & 0.0004 & 0.0041 & 0.0019 & 0.0019 & 0 & 0 & 0 & 0 & 0 & $\ldots$ & 0.0009 & 0.0026 \\
\hline$f_{7}$ & 0 & 0 & 0 & 0 & 0 & 0 & 0.0014 & 0.0004 & 0.0001 & $\ldots$ & 0 & 0 \\
\hline
\end{tabular}


Table 4 The fault trackability of sensors (SST)

\begin{tabular}{|l|c|c|c|c|c|c|c|c|c|c|c|c|}
\hline & A02 & A03 & A04 & A05 & A06 & A07 & A10 & A11 & A12 & $\ldots$ & T25 & T26 \\
\hline$f_{1}$ & 0 & 0 & 0 & 0 & 0 & 0 & 0 & 0 & 0 & $\ldots$ & 0 & 0 \\
\hline$f_{2}$ & 0 & 0 & 0 & 0 & 0 & 0 & 0 & 0 & 0 & $\ldots$ & 0 & 0 \\
\hline$f_{3}$ & 0 & 0 & 0 & 0 & 0 & 0.0047 & 0 & 0 & 0 & $\ldots$ & 0 & 0 \\
\hline$f_{4}$ & 0 & 0 & 0 & 0 & 0 & 0.0047 & 0 & 0 & 0 & $\ldots$ & 0 & 0 \\
\hline$f_{5}$ & 0 & 0 & 0 & 0 & 0 & 0 & 0 & 0 & 0 & $\ldots$ & 0 & 0 \\
\hline$f_{6}$ & 0.0063 & 0.0067 & 0.0062 & 0.0062 & 0 & 0 & 0 & 0 & 0 &.. & 0.0061 & 0.0061 \\
\hline$f_{7}$ & 0 & 0 & 0 & 0 & 0 & 0 & 0.0066 & 0.0022 & 0 & $\ldots$ & 0 & 0 \\
\hline
\end{tabular}

By using SOSM method described in Eq.(1) and considering the testability requirements of the gearbox, the SOSM of the gearbox can be expressed as follows:

$$
\left\{\begin{array}{l}
S S^{*}=\underset{S S}{\arg }\left\{\max \left(\sum_{i}^{7} \sum_{j}^{34} S S T_{i j}\right) \cup \max \left(\sum_{i}^{7} \sum_{j}^{34} S S D_{i j}\right) \min \left(\sum C\left(s s_{i}\right)\right)\right. \\
\text { s.t.FDR } \geq 98 \% ; F I R \geq 94 \% .
\end{array}\right.
$$

where, $\mathrm{SS}=\{\mathrm{A} 02-\mathrm{A} 07, \mathrm{~A} 10-\mathrm{A} 12, \mathrm{~T} 04-\mathrm{T} 27, \mathrm{~T} 31\}$, the number of sensors in SS is 34 and the number of faults in the gearbox is equal to 7, respectively. $\mathrm{SSD}_{i j}$ and $\mathrm{SST}_{i j}$ are listed in Table 3 and 4 , respectively. The main purpose of this paper is to select an optimal sensor set SS* from SS on the basis of meeting the scheduled testability requirements that FDR and FIR are greater than $98 \%$ and $94 \%$, respectively.

The above SOSM is solved using AGASA introduced by tan et al., ${ }^{[6]}$ and the solution process is implemented in MATLAB. The control parameters are initialized as follows: the weight factors of the two objective functions $w_{1}=w_{2}=w_{3}=1 / 3$; the population size PopSize $=40$; the crossover probability $P_{c}=0.95$ and the mutation probability $P_{m}=0.01$; the initial temperature $\mathrm{T}_{0}=100$; the cooling rate $k=0.98$.

The optimized results is $\mathrm{SS}^{*}=\{1,1,1,0,0,0,0,0,0,1,1,1,0,0,0,0,0,0,0,0,0,0,0,0,0,0,0,0,0,0,1,1,0,0\}$, which means that the optimal sensor set is equal to $\{\mathrm{A} 02, \mathrm{~A} 03, \mathrm{~A} 04, \mathrm{~T} 04, \mathrm{~T} 05, \mathrm{~T} 06, \mathrm{~T} 25, \mathrm{~T} 26\}$. And the FDR and FIR of the system with sensor set SS* are $100 \%$ and $96 \%$, respectively. The results show that the testability indices meet the scheduled testability requirements after selecting optimal sensor set SS*, and the sensor costs reduce from 4300 to 1100.

\section{CONCLUSION}

The objective of health monitoring is to obtain high quality information including health condition of a system by means of a lot of sensors. To get more abundant health information, these sensors should have a better ability to detect the fault at its incipient stage and track its development. SOSM proposed in this paper quantifies fault detectability and trackability for each available senor in the gearbox and considers the scheduled testability requirement constraints. Besides, it also can provide an effective way to select a better sensor set with much less cost on the basis of meeting scheduled FDR and FIR for health monitoring. In the future, the study will focus on applying SOSM in complex engineering systems, and a computer-aided design system based on above-mentioned theories is developing to design for sensor selection of health monitoring.

\section{References}

[1] C. S. Byington, T. A. Merdes, and J. D. Kozlowski. "Vibration and Oil Debris Feature Fusion in Gearbox Failures," Proceedings of the 53rd Machinery Failure Prevention Technology Conference, 1999, April.

[2] L. M. Santi, T. S. Sowers, and R. B. Aguilar. "Optimal sensor selection for health monitoring systems". 41st Joint Propulsion Conference and Exhibit, Tucson, United States, 2005, pp. 1-15.

[3] H. Alkhadafe, A. Habaibeh, S. Daihzong and A. Lotfi. "Optimizing Sensor Location for an Enhanced Gearbox Condition Monitoring System”. Journal of physics: conference series, 2012, vol.364, pp.1-9. 
[4] R Leal, J Aguilar, T. Louise et al. "An Approach for Diagnosability Analysis and sensor placement for continuous process based on evolutionary algorithms and analytical redundancy". Applied Mathematical Sciences, 2015, vol.9, pp.2125-2146.

[5] Jung, Daniel. "Diagnosability performance analysis of models and fault detectors". Doctoral thesis. Linköping University, Sweden, 2015.

[6] X. D. Tan, J. Qiu, G. J. Liu, K.H. Lv, S.M. Yang and C. Wang. "A Novel Approach of Testability Modeling and Analysis for PHM Systems Based on Failure Evolution Mechanism”. Chinese Journal of Aeronautics, 2013, vol.26, pp.766-776.

[7] S.Deb, K.R. Pattipati, V.Raghavan, M.Shakeri, and M. Shrestha. "Multi-Signal Flow Graphs: A novel Approach for System Testability Analysis and Fault Diagnosis". IEEE AES Magazine, 1995, vol.10, pp.14-25.

[8] C.D.Begg, T.Merdes, C.Byington, and K Maynard. "Dynamics modeling for mechanical fault diagnostics and prognostics," In: Proceedings of the maintenance and reliability conference, Gatlinburg, Tennessee, 1999, pp. 1-13.

[9] Q. Miao. "Application of Wavelets and Hidden Markov Model in Condition-based Maintenance," University of Toronto, Toronto, 2005.

[10] D. Wang, Q. Miao, and R. Kang. "Robust health evaluation of gearbox subject to tooth failure with wavelet decoposition”. Journal Sound Vibration, 2009, vol. 324(1), pp.1141-57. 